Pacific Journal of Mathematics

ON CONTINUOUS MAPPINGS OF METACOMPACT CzECH

OREL 


\title{
ON CONTINUOUS MAPPINGS OF METACOMPACT ČECH COMPLETE SPACES
}

J. M. WORRELL, JR.

\begin{abstract}
Under what may be thought of as a guise of a description of pathology are indicated here certain ways in which Čech completeness, Arhangel'skiǐ's $p$-space concept, and metacompactness enlarge on the respective concepts of metric absolute $G_{\delta}$ 's, metrizability, and paracompactness. This is done through examination of certain aspects of the theory of multivalued mappings. It is taken as a point of orientation that the topic of Tychonoff locally bicompact spaces has a substantial mathematical interest. It is assumed obvious that such spaces are locally paracompact $p$-spaces. An underlying point of view is that the class of regular locally paracompact $p$-spaces extends along natural lines the class of regular locally metrizable spaces.
\end{abstract}

Let us observe these theorems: (1) A Hausdorff space is paracompact if and only if it is fully normal [13]. (2) A space is metacompact if and only if for every collection $G$ of open sets covering it there exists a collection $H$ of open sets covering it such that if $P$ is a point, the collection of all members of $H$ containing $P$ refines a finite subcollection $K$ of $G$ [22]. (3) A $T_{1}$ space is metrizable if and only if it is fully normal and has a base of countable order (cf. definitions below) [3]. (4) A $T_{1}$ space has a uniform base (cf. definitions below) if and only if it is metacompact and has a base of countable order [27]. (5) Metacompactness is invariant under the action on a topological space of a closed continuous mapping [23]. We may then see that whether or not a metacompact $T_{1}$ topological space $S$ has a perfect mapping onto a space with a uniform base depends only on whether $S$ has a perfect mapping onto a space having a base of countable order. Similarly, since full normalcy of a topological space is also an invariant under the action of a closed continuous mapping [10], whether a fully normal $T_{1}$ space $S$ has a perfect mapping onto a metrizable space depends only on whether $S$ has a perfect mapping onto a space having a base of countable order. These reductions achieve heightened interest in view of the invariance of the base of countable order property under the actions of peripherally bicompact closed continuous mappings on $T_{1}$ spaces [21], the intimacy of its relation to the topic of interior mappings $[16,19]$, and certain work of Frolík and Arhangel'skiî which will now be described.

Frolík showed that a paracompact Hausdorff space is Čech complete (cf. definition below) if and only if it has a perfect mapping 
onto a complete metric space [7]. This fundamental contribution was enlarged by Arhangel'skil , first of all by his exercise of an extraordinary ingenuity in isolating the concept of a $p$-space (cf. definition below), and secondly by his equating for the Hausdorff paracompact cases the property of being a $p$-space with the admitting of a perfect mapping onto a metrizable space [4]. Since Čech completeness is preserved under the action of a perfect mapping between Tychonoff spaces [8], we may now see that both Frolik's theorem and Arhangel'skil's theorem may be interpreted as having in common the remarkable feature of pivoting on the existence of perfect mappings of the respective (paracompact) spaces onto spaces having bases of countable order.

We now observe that the first four theorems reviewed above put one in a position to interpret that for spaces satisfying the first Trennungsaxiom and having uniform bases, the distinction metrizablenonmetrizable corresponds precisely to the distinction unity-finitude of order $>1$ of certain refined collections $K$. Can it be the case, one may ask with this preparation, that nevertheless there exists a metacompact Čech complete space admitting of no perfect mapping onto an absolute $G_{o}$ space with a uniform base or, equivalently (in view of the above theorems), onto a space having a base of countable order? An answer in the negative might be suspected to have profound structural implications.

Let us look at this in another way. H. H. Wicke and the author proposed at the last meeting of the International Congress of Mathematicians in Moscow the thesis that the base of countable order concept, especially when enriched by an appropriate notion of completeability, express substantially much of what is topologically fundamental in the concept of metrizability [18]. If this be valid, then heuristically one might conclude that either every Tychonoff $p$-space has a perfect mapping onto a space having a base of countable order or else there exists a metacompact Tychonoff $p$-space which cannot be so transformed. If the reader but put himself in a frame of mind receptive to this line of reasoning, he may sense a heuristic justification for the either/or conclusion. For radical as it may seem in the contemporary milieu the thesis carries with it the corollary that paracompactness-like properties are not naively a part of the essential content of metrizability. (In this connection, see [14], [17], [20], [24], [25].)

Insofar as technique and exposition can be distinguished in a work of this kind, the technical portion of this mémoire will be devoted to the demonstration of the existence of a first countable, regular $T_{0}$ locally bicompact, screenable, metacompact space $S$ of power $c$ which admits of no Lindelöfian continuous mapping whatsoever onto a Hausdorff space having a base of countable order. It follows that 
$S$ has no perfect mapping onto a space having a base of countable order.

Let us inquire now into the significance of the requirement of first countability of the example. One may say that owing to the definitional sacrifice by any such example of the uniform first countability of the base of countable order property any further interest in first countability is eclipsed to at best a peripheral position of interest. Therefore the underlying issue likely has to do with whether first countability in itself must in certain situations go a long way toward uniformity in this sense. This is in fact the point here. There exist Čech complete spaces $\Sigma$ of ordinal numbers with respect to the order topology which have no perfect mappings onto spaces with bases of countable order. But every first countable subspace of such a $\Sigma$ has a base of countable order since it contains no dense subspace [20]. The significance of this is reinforced by the behavior of the base of countable order property under the action of Cartesian products [15] and its hereditary character [27].

One might pursue the significance of the existence of such examples in considerable additional detail. Why the emphasis on the power of the space? Why the reference to $\sigma$-discrete refinements? Why the specific mention of screenability? Certain of these questions bear on the intimacy of the interplay between the topics of bases of countable order and paracompactness-like properties [22]. Suffice it here to say that it was felt virtually obligatory to resolve the above question prior to stating the general thesis [28, cf. also 17].

2. Definitions and notation. Except that the null set convention is not employed, general terminology usually follows along the lines of [9]. As a technical point it is noted that compactness is taken in the Fréchet sense, though in the present context one might apply the theorem of [2] that $T_{1}$ compact metacompact spaces are bicompact. For screenable space and development of a space, see [5]; for metacompact space, see [2]. As in [11], if $K$ is a collection of sets, $K^{*}$ denotes the sum of the elements of $K$. If $M$ is a point set, $\bar{M}$ denotes the (contextually implicit) closure of $M$. By an arc will be understood a bicompact connected Hausdorff point set having exactly two noncut points. By an endpoint of an arc $\alpha$ is meant a noncut point of $\alpha$. A perfect mapping is a bicompact, closed continuous mapping. A uniform base for a space $S$ is a base $B$ for $S$ such that if $B^{\prime}$ is an infinite subcollection of $B$ and $P$ belongs to all members of $B^{\prime}$, then $B^{\prime}$ is a base for $S$ at $P$ [1]. Note that a developable space has a uniform base if and only if it is metacompact [1]. 
A space is Čech complete if and only if it is a Tychonoff space $S$ the set of all points of which is an inner limiting set in a StoneCech bicompactification $\beta(S)$ [6]. It follows that the set of all points of $S$ is an inner limiting set of any Hausdorff space in which $S$ can be densely embedded [6]. Note that all regular $T_{0}$ locally bicompact spaces are Cech complete. A $T_{1}$ space $S$ is a $p$-space if and only if it is covered by each term of a sequence $G_{1}, G_{2}, \ldots$ of collections of open sets of a Wallman bicompactification $\omega S$ such that for each point $P$ of $S$, all points common to the sets $s t\left(G_{n}\right)_{P}$ belong to $S$ [4]. For the Tychonoff cases, this requires such a sequence $G_{1}, G_{2}, \cdots$ with respect to $S$ for any Hausdorff space in which $S$ can be densely embedded. Note the analogy with developability [cf. 26].

A base of countable order for a space $S$ is a base $B$ for $S$ such that if $D_{1}, D_{2}, \ldots$ is a sequence of distinct members of $B$ each including its successor and $P$ is a point common to all the sets $D_{n}$, then $\left\{D_{1}\right\}+\left\{D_{2}\right\}+\cdots$ is a base for $S$ at $P$ [3]. Particularly to be noted are the role of bases of countable order in the characterization of developability involving a paracompactness-like refinement condition [27], the close bearing of the concept on the topic of interior transformations $[16,19]$, and its tractability to appropriate completeness formulations $[18,19,20]$.

3. The construction. The technique of construction utilizes in a rather straightforward manner classical theorems on transfinite cardinalities of a sort such as are developed in [12]. The proof of properties is designed to reduce the question of the existence of certain Lindelöfian mappings in effect to that of the existence of a perfect mapping onto a space having a base of countable order through utilization of restrictions to certain bicompact domains.

THEOREM. There exists a metacompact screenable locally compact Hausdorff space $S$ of the power of the continuum satisfying these conditions: (1) Any collection of open sets covering $S$ is refined by a $\sigma$ discrete collection of point sets covering $S$. (2) No Lindelöfian continuous mapping with $S$ as its domain has a Hausdorff space with a base of countable order as its range. (3) $S$ is first countable.

Proof. There exists a sequence $\alpha_{1}, \alpha_{2}, \cdots$ of mutually exclusive first countable arcs of cardinal number $c$ such that for each $n$, there exists a collection $Q_{n}$ of mutually exclusive subarcs of $\alpha_{n}$ satisfying these conditions: (1) No element of $Q_{n}$ contains an endpoint of $\alpha_{n}$. (2) $Q_{n}^{*}$ is dense in $\alpha_{n}$. (3) If $q$ and $q^{\prime}$ are two elements of $Q_{n}$ then (a) there exists a nonseparable subset $Y$ of $\alpha_{n}-Q_{n}^{*}$ such that $q$ 
separates $Y$ from one endpoint of $\alpha_{n}$ (in the sense of [11]) and $q^{\prime}$ separates $Y$ from the other and (b) there exist $c$ members of $Q_{n}$ similarly separated from the endpoints of $\alpha_{n}$ by $q$ and $q^{\prime}$.

Let $\Gamma$ denote the set of all sequences $J_{1}, J_{2}, \ldots$ such that each $J_{n}$ is a subarc of $\alpha_{n}$ the endpoints of which belong to $\alpha_{n}-Q_{n}^{*}$. Let $M$ denote a set of power $c$ not intersecting $\alpha_{1}+\alpha_{2}+\cdots$. There exists a transformation $\theta$ of $M$ onto a collection $W$ of simple infinite sequences such that (1) the $n^{\text {th }}$ term of each sequence in $W$ belongs to $Q_{n}$, (2) no element of $Q_{1}+Q_{2}+\cdots$ is a term of two members of $W$, and (3) if $J_{1}, J_{2}, \cdots$ belongs to $\Gamma$, there exist $c$ sequences $q_{1}, q_{2}, \cdots$ in $W$ such that each $J_{n}$ includes $q_{n}$. For each $q$ in $Q_{1}+Q_{2}+\cdots$, let $X_{q}$ denote a cut point of $q$. For each $n$, let $\alpha_{n}^{\prime}$ denote the set of all points $P$ of $\alpha_{n}$ such that either (1) $\alpha-Q_{n}^{*}$ contains $P$ or (2) $P$ is a noncut point of some member of $Q_{n}$ or (3) $P$ is $X_{q}$ for some $q$ in $Q_{n}$.

Let $\tau$ denote the collection to which an element belongs if and only if it is the sum of some sets $D$ satisfying one of these conditions:

(1) For some $n, D$ is an open set of $\alpha_{n}^{\prime}$ (in the relative topology).

(2) For some $n$ and element $\mu$ of $M, D$ is

$$
\{\mu\}+\left\{X_{q_{n}}\right\}+\left\{X_{q_{n+1}}\right\}+\cdots,
$$

where $q_{1}, q_{2}, \cdots$ denotes $\theta(\mu)$. Let $S$ denote $\tau^{*}$.

The first countable regular $T_{0}$ space $(S, \tau)$ is screenable, locally compact, and has the $\sigma$-discrete refinement property stated in the theorem. All regular spaces with this $\sigma$-discrete refinement property are countably metacompact. Moreover, every countably metacompact screenable space is metacompact. Hence $(S, \tau)$ is metacompact. Since $(S, \tau)$ is a regular $T_{0}$ locally bicompact space, it is Čech complete. Clearly, $\overline{\bar{S}}=c$.

Suppose there exists a Lindelöfian continuous mapping $f$ of $(S, \tau)$ onto a Hausdorff space having a base of countable order.

(I) Each $f / \alpha_{n}^{\prime}$ is closed and bicompact. Since having a base of countable order is an hereditary property for a space [27], $f\left(\alpha_{n}^{\prime}\right)$ has such a base [21]. Thus the bicompact Hausdorff space $f\left(\alpha_{n}^{\prime}\right)$ is metrizable [3].

For some $n$ let $G$ denote the decomposition of $\alpha_{n}^{\prime}$ induced by $f$. There exists a meaning for the notation $U_{i, h}$, for positive integers $i$ and subsets $h$ of $G$, such that for some development $H_{1}, H_{2}, \cdots$ of $G$ (with respect to the quotient topology) the terms of which are finite, these conditions are satisfied: (1) For each $i$ and element $h$ of $H_{i}, U_{i, h}$ is a finite collection of sets covering $h^{*}$ any nondegenerate element of which is the common part of $\alpha_{n}^{\prime}$ and some connected open 
subset of $\alpha_{n}$. (2) For each $i$ and element $h$ of $H_{i+1}$ there exists some $h^{\prime}$ in $H_{i}$ including $h$ such that the closure of each member of $U_{i+1, h}$ is a subset of some member of $U_{i, h^{\prime}}$ and is covered by $h^{\prime}$. For each $i$, let $K_{i}$ denote the sum of all collections $U_{i, h}$ for elements $h$ of $H_{i}$.

With application of König's lemma it may be seen that if $P$ belongs to $\alpha_{n}-Q_{n}^{*}$ and $g$ is the member of $G$ containing $P$ there exist sequences $h_{1}, h_{2}, \ldots$ and $D_{1}, D_{2}, \ldots$ of sets such that (1) each $h_{i}$ belongs to $H_{i}$, contains $g$, and includes $\bar{h}_{i+1}$, (2) each $D_{i}$ is a member of $U_{k, h_{i}}$ containing $P$, and (3) each $D_{i}$ includes $\bar{D}_{i+1}$. With use of the compactness of $\alpha_{n}^{\prime}$ it may be seen that if $\{P\}$ is the common part of the sets $D_{i}$ then $\left\{D_{1}\right\}+\left\{D_{2}\right\}+\cdots$ is a base for $\alpha_{n}^{\prime}$ at $P$. Since $K_{1}+K_{2}+\cdots$ is countable and $\alpha_{n}-Q_{n}^{*}$ is nonseparable, there exist some $P$ in $\alpha_{n}-Q_{n}^{*}, h_{1}, h_{2}, \cdots$ and $D_{1}, D_{2}, \cdots$ as above such that the common part $L$ of the sets $\bar{D}_{i}$ is nondegenerate. With use of conditions (2) and (3) of the first paragraph of this proof it may be seen that $L$ contains two points of $\alpha_{n}-Q_{n}^{*}$. Since $H_{1}, H_{2}, \cdots$ is a development for $G$ and each $h_{i}$ includes $\bar{h}_{i+1}$, it may be seen that $h_{1}^{*}, h_{2}^{*}, \cdots$ converges to the member $g$ of $G$ containing $P$. This requires that $L$ be a subset of $g$.

(II) Let $\Delta$ denote the decomposition of $S$ induced by $f$. Suppose there exist a member $\delta$ of $\Delta$ and a sequence $n_{1}, n_{2}, \cdots$ of increasing positive integers such that for each $i, \delta$ includes the common part of $S$ and some subarc $v_{i}$ of $\alpha_{n_{i}}$ the endpoints of which belong to $\alpha_{n_{i}}-Q_{n_{i}}^{*}$. Then there exists a sequence $J_{1}, J_{2}, \cdots$ belonging to $\Gamma$ such that for each $i, J_{n_{i}}$ is $v_{i}$. With the use of condition (3) of the definition of $\theta$ and the definition of $\tau$ it may be seen that there exists an uncountable closed and isolated subset of $M$ which is on the boundary of

$$
S \cdot\left(v_{1}+v_{2}+\cdots\right)
$$

and which therefore must be included by the closed point set $\delta$. But this involves a contradiction, for $\delta$ is Lindelöfian. With application of (I) above it follows that there exist a sequence $\delta_{1}, \delta_{2}, \cdots$ of distinct members of $\Delta$ and a sequence $n_{1}, n_{2}, \cdots$ of increasing positive integers suchthat for each $i, \alpha_{n_{i}}$ has a subarc $v_{i}$ the endpoints of which belong to $\alpha_{n_{i}}-Q_{n_{i}}^{*}$ such that $\delta_{i}$ includes $v_{i} \cdot \alpha_{n_{i}}^{\prime}$. Since no $\delta_{i}$ contains uncountably many elements of $M$, there exists a countable subset $T$ of $M$ such that $\delta_{1}+\delta_{2}+\cdots$ does not intersect $M-T$. Uncountably many points of $M$ belong to the boundary of $S \cdot\left(v_{1}+v_{2}+\cdots\right)$. So there exists an element $\delta$ of $\Delta$ intersecting $M-T$ and an infinite subsequence $\sigma$ of $\delta_{1}, \delta_{2}, \cdots$ such that $f(\sigma)$ converges uniquely to $\delta$. But a contradiction is involved, for $T+\delta \cdot M$ is countable, and uncountably many points of $M$ are limit points of the sum of the terms of $\sigma$.

It follows that there exists no Lindelöfian continuous mapping of $(S, \tau)$ onto a Hausdorff space having a base of countable order. 


\section{REFERENCES}

1. P. S. Alexandroff, On the metrization of topological spaces, Bull. Acad. Polon. Sci., Sér. Sci. Math., Astr. Phys. 8 (1960), 135-140 (in Russian).

2. R. Arens and J. Dugundji, Remark on the concept of compactness, Portugal. Math. 9 (1950), 141-143.

3. A. V. Arhangel'skil, Certain metrization theorems, Uspehi Mat. Nauk 18 (1963), 139-145 (in Russian).

4. - On a class of spaces containing all metric and all locally bicompact spaces, Mat. Sb. 67 (1965), 55-85 (in Russian).

5. R. H. Bing, Metrization of topological spaces, Canad. J. Math. 3 (1951), 175-186.

6. E. Čech, On bicompact spaces, Ann. of Math. 38 (1937), 823-844.

7. Z. Frolík, On the topological product of paracompact spaces, Bull. Acad. Polon. Sci., Sér. Sci. Math., Astr. Phys. 8 (1960), 747-750.

8. - Locally $G_{\delta}$-spaces, Czech. Math. J. 12 (87) (1962), 346-355.

9. J. L. Kelley, General topology, Van Nostrand, New York, 1955.

10. E. Michael, Another note on paracompact spaces, Proc. Amer. Math. Soc. 8 (1957), 822-828.

11. R. L. Moore, Foundations of point set theory, rev. ed., Amer. Math. Soc. Colloq. Publ. 13, Amer. Math. Soc., Providence, R. I., 1962.

12. W. Sierpiński, Cardinal and ordinal numbers, second edition revised, Warsaw, 1965.

13. A. H. Stone, Paracompactness and product spaces, Bull. Amer. Math. Soc. 54 (1948), 977-982.

14. H. H. Wicke, Concerning an arc theorem, Notices Amer. Math. Soc. 14 (1967), 290.

15. - Product mappings and Cartesian products of spaces having bases of countable order, Notices Amer. Math. Soc. 14 (1967), 424.

16. - The regular open continuous images of complete metric spaces, Pacific J. Math. 23 (1967), 621-625.

17. - On a theory of topological structure involving principles of uniformization, Kempner Colloquium Address, University of Colorado, 1967. (To appear in expanded from with J. M. Worrell, Jr., as a Sandia Corporation Research Report.)

18. H. H. Wicke and J. M. Worrell, Jr., Open continuous mappings and bases of countable order, Presented by $\mathrm{H}$. H. Wicke at the International Congress of Mathematicians, Moscow, 1966. Abstract published in Abstracts of Brief Scientific Communications, International Congress of Mathematicians, Moscow, 1966, p. 14.

19. - Open continuous mappings of spaces having bases of countable order, Duke Math. J. 34 (1967), 255-272.

20. - On completeness of first countable Hausdorff spaces (manuscript submitted). Presented by H. H. Wicke as an invited address, May 1968, University of New Mexico.

21. J. M. Worrell, Jr., Decompositions of spaces having bases of countable order, Notices Amer. Math. Soc. 11 (1964), 773-774. (Certain portions of the material to appear in Upper semicontinuous decompositions of spaces having bases of countable order, Portugal. Math.).

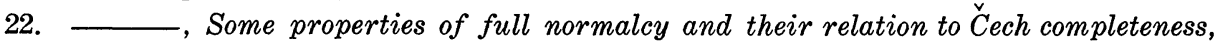
Notices Amer. Math. Soc. 14 (1967), 555. (A part of this material has appeared in the author's article, A characterization of metacompact spaces, Portugal. Math. 25 (1966), 171-174.)

23. - The closed continuous images of metacompact topological spaces, Portugal. Math. 25 (1966), 175-179. 
24. - Completeness and certain implicit upper semi-continuous decompositions, Notices Amer. Math. Soc. 12 (1965), 378.

25. - Concerning a theorem of Lavrentieff, Notices Amer, Math. Soc. 14 (1967), 161.

26. Closed continuous mapping of p-spaces, Notices Amer. Math. Soc. 14 (1967), 856.

27. J. M. Worrell, Jr., and H. H. Wicke, Characterizations of developable topological spaces, Canad. J. Math. 17 (1965), 820-830.

28. - Non-first-countable topological structure, Notices Amer. Math. Soc. 14 (1967), 935.

Received February 7, 1968. This work was supported by the United States Atomic Energy Commission. Preliminary work in the identification and isolation of the problem was supported by a National Science Foundation Postdoctoral Fellowship.

SANDIA LABORATORIES

Albuquerque, NeW Mexico 


\title{
PACIFIC JOURNAL OF MATHEMATICS
}

\author{
EDITORS
}

H. ROYDEN

Stanford University

Stanford, California

R. R. PhelPS

University of Washington

Seattle, Washington 98105
J. DUGUNDJI

Department of Mathematics

University of Southern California

Los Angeles, California 90007

RICHARD ARENS

University of California

Los Angeles, California 90024

\section{ASSOCIATE EDITORS}
E. F. BECKENBACH
B. H. NeumanN
F. WOLF
K. YoSHIDA

\section{SUPPORTING INSTITUTIONS}

\author{
UNIVERSITY OF BRITISH COLUMBIA \\ CALIFORNIA INSTITUTE OF TECHNOLOGY \\ UNIVERSITY OF CALIFORNIA \\ MONTANA STATE UNIVERSITY \\ UNIVERSITY OF NEVADA \\ NEW MEXICO STATE UNIVERSITY \\ OREGON STATE UNIVERSITY \\ UNIVERSITY OF OREGON \\ OSAKA UNIVERSITY \\ UNIVERSITY OF SOUTHERN CALIFORNIA
}

\author{
STANFORD UNIVERSITY \\ UNIVERSITY OF TOKYO \\ UNIVERSITY OF UTAH \\ WASHINGTON STATE UNIVERSITY \\ UNIVERSITY OF WASHINGTON \\ $\stackrel{*}{*} \stackrel{*}{*}{ }^{*}{ }^{*}$ \\ CHEVRON RESEARCH CORPORATION \\ TRW SYSTEMS \\ NAVAL WEAPONS CENTER
}

The Supporting Institutions listed above contribute to the cost of publication of this Journal, but they are not owners or publishers and have no responsibility for its content or policies.

Mathematical papers intended for publication in the Pacific Journal of Mathematics should be in typed form or offset-reproduced, double spaced with large margins. Underline Greek letters in red, German in green, and script in blue. The first paragraph or two must be capable of being used separately as a synopsis of the entire paper. It should not contain references to the bibliography. Manuscripts, in duplicate if possible, may be sent to any one of the four editors. Please classify according to the scheme of Math. Rev. 36, 1539-1546. All other communications to the editors should be addressed to the managing editor, Richard Arens, University of California, Los Angeles, California, 90024.

50 reprints are provided free for each article; additional copies may be obtained at cost in multiples of 50 .

The Pacific Journal of Mathematics is published monthly. Effective with Volume 16 the price per volume (3 numbers) is $\$ 8.00$; single issues, $\$ 3.00$. Special price for current issues to individual faculty members of supporting institutions and to individual members of the American Mathematical Society: $\$ 4.00$ per volume; single issues $\$ 1.50$. Back numbers are available.

Subscriptions, orders for back numbers, and changes of address should be sent to Pacific Journal of Mathematics, 103 Highland Boulevard, Berkeley, California, 94708.

PUBLISHED BY PACIFIC JOURNAL OF MATHEMATICS, A NON-PROFIT CORPORATION

Printed at Kokusai Bunken Insatsusha (International Academic Printing Co., Ltd.), 7-17, Fujimi 2-chome, Chiyoda-ku, Tokyo, Japan. 


\section{Pacific Journal of Mathematics \\ Vol. 30, No. $2 \quad$ October, 1969}

Gregory Frank Bachelis, Homomorphisms of annihilator Banach algebras.

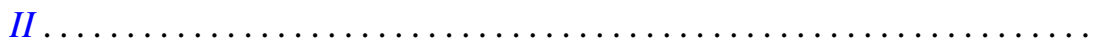

Leon Bernstein and Helmut Hasse, An explicit formula for the units of an algebraic number field of degree $n \geq 2 \ldots \ldots \ldots \ldots \ldots \ldots \ldots . \ldots 29$

David W. Boyd, Best constants in a class of integral inequalities ........ 367

Paul F. Conrad and John Dauns, An embedding theorem for lattice-ordered

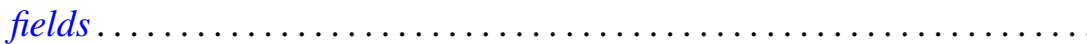

H. P. Dikshit, Summability of Fourier series by triangular matrix

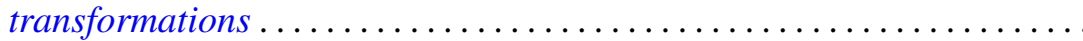

Dragomir Z. Djokovic, Linear transformations of tensor products preserving a fixed rank............................. 411

John J. F. Fournier, Extensions of a Fourier multiplier theorem of Paley . . . 415 Robert Paul Kopp, A subcollection of algebras in a collection of Banach

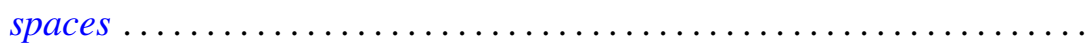

Lawrence Louis Larmore, Twisted cohomology and enumeration of vector bundles ...................................... 437

William Grenfell Leavitt and Yu-Lee Lee, A radical coinciding with the lower radical in associative and alternative rings .................

Samuel Merrill and Nand Lal, Characterization of certain invariant subspaces of $H^{p}$ and $L^{p}$ spaces derived from logmodular

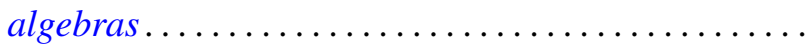

Sam Bernard Nadler, Jr., Multi-valued contraction mappings ....

T. V. Panchapagesan, Semi-groups of scalar type operators in Banach spaces ....................................

J. W. Spellmann, Concerning the infinite differentiability of semigroup motions

H. M. (Hari Mohan) Srivastava, A note on certain dual series equations involving Laguerre polynomials.

Ernest Lester Stitzinger, A nonimbedding theorem of associative algebras................................

J. Jerry Uhl, Jr., Martingales of vector valued set functions ...

John Mays Worrell Jr., On continuous mappings of metacompact $\check{C} e c h$

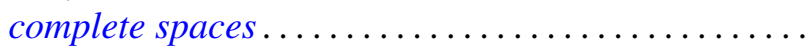

